\title{
Cold dust clumps in dynamically hot gas ${ }^{\star}$
}

\author{
S. Kim ${ }^{1}$, E. Kwon ${ }^{1}$, S. C. Madden², M. Meixner ${ }^{3,19}$, S. Hony ${ }^{2}$, P. Panuzzo ${ }^{2}$, M. Sauvage ${ }^{2}$, J. Roman-Duval ${ }^{3}$, \\ K. D. Gordon ${ }^{3}$, C. Engelbracht ${ }^{4}$, F. P. Israel ${ }^{5}$, K. Misselt ${ }^{4}$, K. Okumura ${ }^{2},{\mathrm{~A} . \mathrm{Li}^{6}}^{6}$, A. Bolatto ${ }^{7}$, R. Skibba ${ }^{4}$, F. Galliano ${ }^{2}$, \\ M. Matsuura ${ }^{8,9}$, J.-P. Bernard ${ }^{10}$, C. Bot ${ }^{11}$, M. Galametz ${ }^{2}$, A. Hughes ${ }^{12,13}$, A. Kawamura ${ }^{14}$, T. Onishi ${ }^{15}$, D. Paradis ${ }^{16}$, \\ A. Poglitsch ${ }^{17}$, W. T. Reach ${ }^{16,18}$, T. Robitaille ${ }^{19}$, M. Rubio ${ }^{20}$, and A. G. G. M. Tielens ${ }^{5}$
}

(Affiliations can be found after the references)

Received 31 March 2009 / Accepted 16 April 2010

\begin{abstract}
Aims. We present clumps of dust emission from Herschel observations of the Large Magellanic Cloud (LMC) and their physical and statistical properties. We catalog cloud features seen in the dust emission from Herschel observations of the LMC, the Magellanic type irregular galaxy closest to the Milky Way, and compare these features with H I catalogs from the ATCA+Parkes H I survey.

Methods. Using an automated cloud-finding algorithm, we identify clouds and clumps of dust emission and examine the cumulative mass distribution of the detected dust clouds. The mass of cold dust is determined from physical parameters that we derive by performing spectral energy distribution fits to 250,350 , and $500 \mu \mathrm{m}$ emission from SPIRE observations using dust grain size distributions for graphite/silicate in low-metallicity extragalactic environments.

Results. The dust cloud mass spectrum follows a power law distribution with an exponent of $\gamma=-1.8$ for clumps larger than $4 \times 10^{2} M_{\odot}$ and is similar to the H I mass distribution. This is expected from the theory of ISM structure in the vicinity of star formation.
\end{abstract}

Key words. submillimeter: ISM - Magellanic Clouds - ISM: structure - ISM: clouds

\section{Introduction}

The overall structure and evolution of the ISM of a galaxy is determined by the physical and dynamical conditions in the diffuse ISM where most of the processing of gas and dust clouds occurs. Thus inspecting the cloud properties by studying the atomic gas and the dust emission under different environmental conditions will help us to understand how this processing of matter proceeds.

In the present paper, we study the dynamical conditions of the diffuse gas in the Large Magellanic Cloud (LMC). We examine optically thin dust emission at far-infrared (FIR) and sub-millimeter wavelengths by studying the spectral energy distributions (SEDs) inferred from radiative transfer models. By combining an H I synthesis survey and an imaging survey in the FIR and submillimeter emission performed by Herschel Space Observatory (Pilbratt et al. 2010), we investigate the detailed relationship between the diffuse atomic gas and interstellar dust emission in the LMC. The Herschel Space Observatory is designed to study the submillimeter and FIR emission from our universe and offers unprecedented resolution. The Herschel has a payload of two cameras, PACS (Poglitsch et al. 2010) and SPIRE (Griffin et al. 2010). PACS employs two bolometer arrays for imaging, and SPIRE comprises spider-web bolometers

* Herschel is an ESA space observatory with science instruments provided by European-led Principal Investigator consortia and with important participation from NASA. with NTD Ge temperature sensors. Mapping strategy and data processing procedures are described in detail by Meixner et al. (2010).

The Herschel survey of the Magellanic Clouds will provide information about the interstellar dust emission (Meixner et al. 2006) from all phases of the ISM in the Magellanic Clouds. For diffuse atomic gas, we use data from the H I aperture synthesis survey in Kim et al. (1998) and data from the combined survey using single-dish observations with the Parkes 64-m radio telescope (Staveley-Smith et al. 2003) that was published in Kim et al. (2003). The SED models provide spectral information in the $160 \mu \mathrm{m}$ to $500 \mu \mathrm{m}$ wavelength range. Relatively optically thin dust emission at FIR and submillimeter wavelengths can also provide information about the gas mass in the ISM because the gas and dust are well mixed in most of the ISM phases. Therefore, the dust emission at the FIR and submillimeter wavelengths can trace the column density and the structure of the ISM.

\section{Cold dust associated with atomic hydrogen}

\subsection{Dust clumps}

The unprecedented quality of the SPIRE images improves our knowledge of the spatial distribution of galactic infrared/submillimeter dust radiation and characterizes it as cloud features with clumps and filaments. We adopt the "clump" terminology to represent entities with properties of typical "clouds" 

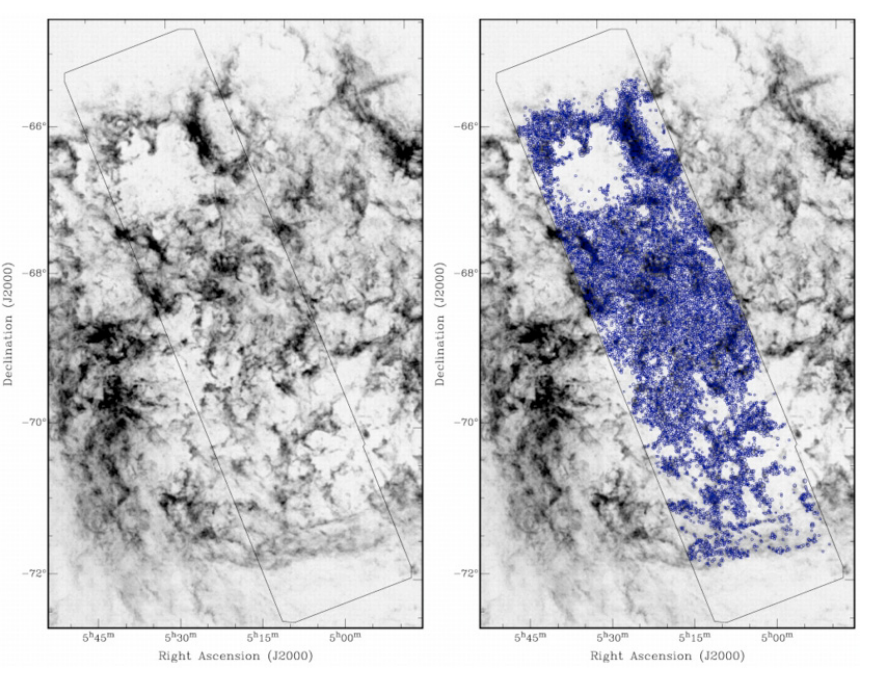

Fig. 1. Dust clouds seen in the SPIRE $500 \mu \mathrm{m}$ image were identified using an automatic finding algorithm in which the lowest threshold was set to be $3 \sigma$ (blue contours), and they are overlaid on the $\mathrm{HI}$ aperture synthesis image (Kim et al. 2003) of the LMC. $1 \sigma$ is set to $0.3 \mathrm{MJy} / \mathrm{sr}$ at $500 \mu \mathrm{m}$. The black box indicates the observed region in the Science Demonstration Program of Herschel Space Observatory (Meixner et al. 2010).

seen in the molecular clouds in the Galaxy (Bergin \& Tafalla 2007). We examine the brightness distribution of the pixels treating them as clumps by searching for peaks of local emission and adding the neighboring pixels of one clump to the objects (Williams et al. 1994). We find 7449 dust clumps generated by an automatic clump-finding routine for the $500 \mu \mathrm{m}$ emission. The threshold being set to three times of an rms of $\sim 0.3 \mathrm{MJy} / \mathrm{sr}$ (Fig. 1). The automatic clump-finding algorithm determines structure by first contouring the data at a multiple of the rms noise of the data, searching for peaks of emission that are local maxima in the SPIRE images, and then connecting pixels at each contour level from the highest to lower intensities. Isolated contours at each level are identified as clumps (Williams et al. 1994).

By applying the same method to the $350 \mu \mathrm{m}$ image of the LMC, we find 8460 dust clumps (Fig. 2). More faint clumps are seen at $350 \mu \mathrm{m}$ than $500 \mu \mathrm{m}$ above the noise level. However, the dust clumps at $500 \mu \mathrm{m}$ are well correlated with the $\mathrm{H}$ I clouds and filaments. In general, the dust clumps at $500 \mu \mathrm{m}$ identified in the present study are distributed quite uniformly in the LMC. Thus, for the clump analysis pursued in this study we use the clumps identified in the $500 \mu \mathrm{m}$ image.

For a clump consisting of a set of pixels with positions $\{x, y\}$ and intensity $\{I\}$, the size of each clump is calculated based on its extent in the spatial dimension below. The clumps range in size from 9.8 to $47 \pm 1 \mathrm{pc}$ with a median of $15 \pm 1 \mathrm{pc}$ in radius given by

$\sigma_{x}^{2}=\left[\frac{\sum I x^{2}}{\sum I}-\left(\frac{\sum I x}{\sum I}\right)^{2}\right]$.

\subsection{Physical properties of dust clumps}

Each dust clump was characterized by the radiation source properties associated with the dust clump and their ambient medium using DUSTY radiative transfer calculations performed by Ivezić et al. (1999). This model assumes a spherical geometry and supports an analytical form of the dust density
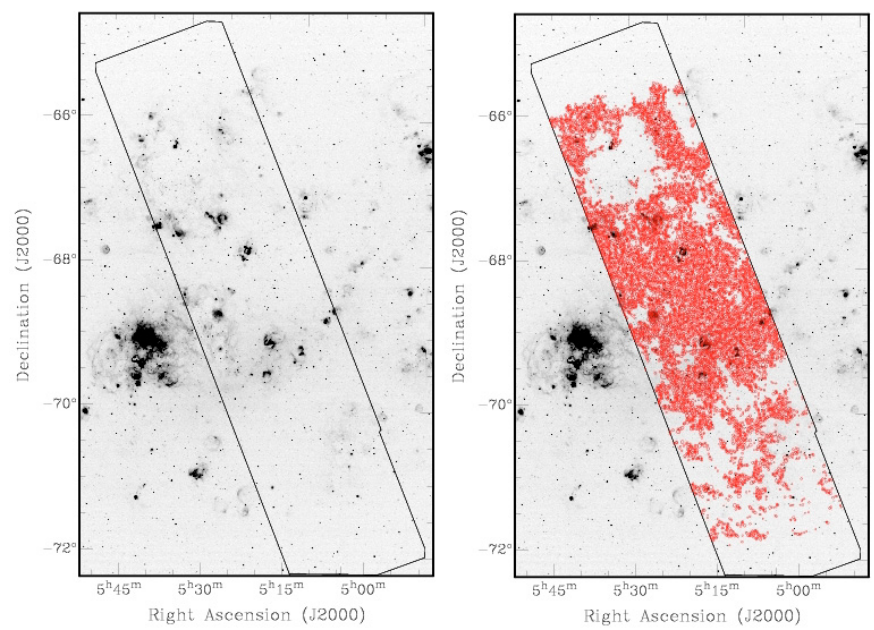

Fig. 2. Dust clouds seen in the SPIRE $350 \mu \mathrm{m}$ image were identified using an automatic finding algorithm, the lowest threshold being set to $3 \sigma$ (red contours), and overlaid on the $\mathrm{H} \alpha$ image of the LMC (Kim et al. 1999). The black box indicates the observed region in the Science Demonstration Program of Herschel Space Observatory (Meixner et al. 2010).

distribution (Sarkar et al. 2006). The physical parameters constrained by fitting the observed SEDs include the chemical composition of the dust, grain size distribution, dust temperature at the inner and outer boundary, dust density distribution, optical depth at a specific wavelength, and the ambient interstellar radiation field (ISRF). We adopted the chemical properties of the dust determined by Draine \& Li (2001) and Weingartner \& Draine (2001). For dust in the neutral medium, we adopted the size distribution of Kim et al. (1994, KMH) using the power law distribution function for grain sizes, $n(a) \propto a^{-q} e^{-a / a_{0}}$ with $q=3.5$, $a_{\min }=0.005 \mu \mathrm{m}$, and $a_{\max }=0.25 \mu \mathrm{m}$. The classical model of grain size distribution was constructed by Mathis et al. (1977, MRN), and Draine \& Lee (1984) revised the MRN model with dielectric functions for graphite and silicate (Draine \& Li 2001).

Interstellar grain temperatures are calculated from

$$
\int_{0}^{\infty} \pi B_{\lambda}\left(T_{d}\right) Q_{\mathrm{IR}}(a, \lambda) \mathrm{d} \lambda=\int_{912 \AA}^{\infty} F_{\lambda} Q_{\mathrm{UV}}(a, \lambda) \mathrm{d} \lambda
$$

where $B_{\lambda}(T)$ is the Planck function for dust at temperature $T$, $F_{\lambda}$ is the flux of the interstellar radiation field, and $Q_{\mathrm{IR}}(a, \lambda)$ and $Q_{\mathrm{UV}}(a, \lambda)$ indicate the infrared and ultraviolet absorption efficiencies, respectively, and are functions of the dust grain radius $a$ (Spitzer 1978). The ISRF is produced by three components: (1) emission from stars; (2) dust emission in the far-IR associated with the heating of interstellar matter by absorption of star light; and (3) the cosmic background radiation (Cox et al. 1986; Chi \& Wolfendale 1991; Strong et al. 2000). Unlike the Milky Way, confusion along the line-of-sight to the LMC is negligible because of its low inclination (Weinberg \& Nikolaev 2001). The LMC also has low internal reddening, and its proximity permits the detailed study of its stellar population. Therefore, the ISRF in the LMC can be estimated with confidence.

The dust equilibrium temperatures are obtained by determining the energy balance between absorption and emission. In Fig. 3, we present three examples of applying DUSTY to different clumps. The result of each fitting provides a characteristic dust emission temperature, $T_{\mathrm{d}}$ for each clump and an estimate of the graphite and silicate abundance ratio. The resulting graphite and silicate abundances from fitting the observed SEDs differ by $50 \%$ among the clumps in different environments in the 

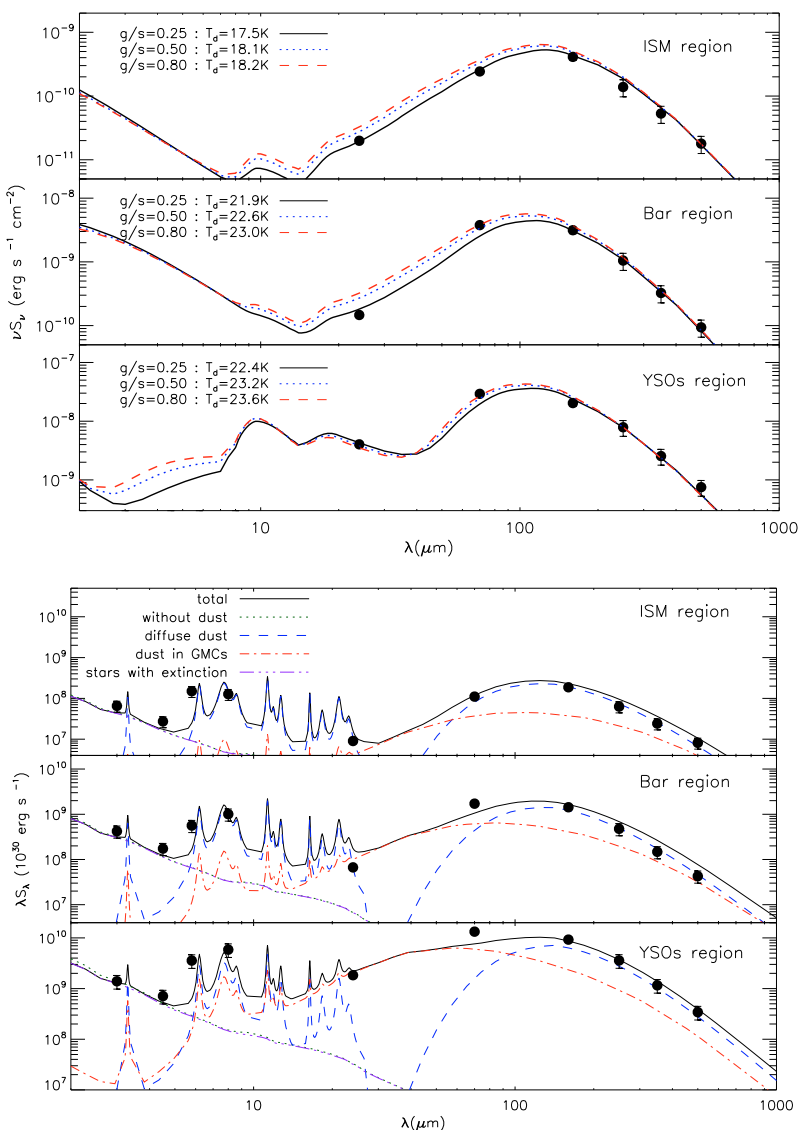

Fig. 3. DUSTY model spectra and the best SED fitting results for three representative dust clumps in different environments (Top). The ISRF for each clump is $1.0 G_{0}$ (ISM region), $3.5 G_{0}$ (Bar region), and $7.0 G_{0}$ (YSOs region). GRASIL (Silva et al. 1998) model spectra and the observed SEDs (Bottom). The data points are from IRAC, MIPS, and SPIRE.

LMC. The second approach to characterize clump temperature is to apply GRASIL (Silva et al. 1998). This spectrophotometric self-consistent model computes the absorption and emission by dust in three different environments such as molecular clouds, diffuse ISM, and AGB envelopes with a dust model consisting of big grains, small grains, and polycyclic aromatic hydrocarbons (PAH) molecules. The consistency check on the equilibrium temperature is made using the GRASIL model and the observed SEDs (Fig. 3). In general, the observed fluxes at $160-500 \mu \mathrm{m}$ are ascribed to the dust in the diffuse medium heated by the interstellar radiation field. Heating of diffuse gas in the ISM can occur by means of the photoelectric heating in interstellar clouds where the dust grains act as catalytic surfaces. The resultant temperature from the fit to the observed SEDs ranges from $15 \pm 0.4$ to $25 \pm 0.4 \mathrm{~K}$ and is similar to the SPIRE temperature map (Gordon et al. 2010).

At a given grain temperature, the mass of each dust clump is calculated from $M_{\mathrm{d}}=S_{\lambda} \times D^{2} / \kappa_{\lambda} \times B_{\lambda}\left(T_{\mathrm{d}}\right)$, where $S_{\lambda}$ is the flux density at $500 \mu \mathrm{m}$ and $D$ is the distance of the galaxy at $50 \mathrm{kpc}$ (Schaefer 2008), and $B_{\lambda}\left(T_{\mathrm{d}}\right)$ is the value of the Planck function at $500 \mu \mathrm{m}$, and a function of $T_{\mathrm{d}}$. We adopt a mean temperature for each clump as a function of the three categories shown in Fig. 3. The YSOs region and bar region in Fig. 3 indicate dynamically hot regions described in Kim et al. $(1998,2007)$, where $\kappa_{\lambda}$ is the mass absorption coefficient at $500 \mu \mathrm{m}$ from the SPIRE observations and $\kappa_{\lambda} \sim 1.15 \mathrm{~cm}^{2} / \mathrm{g}$ is used. The derived dust masses are in the range of $1.8 \times 10^{1} M_{\odot}<M_{\text {dust }}<7.9 \times 10^{3} M_{\odot}$.
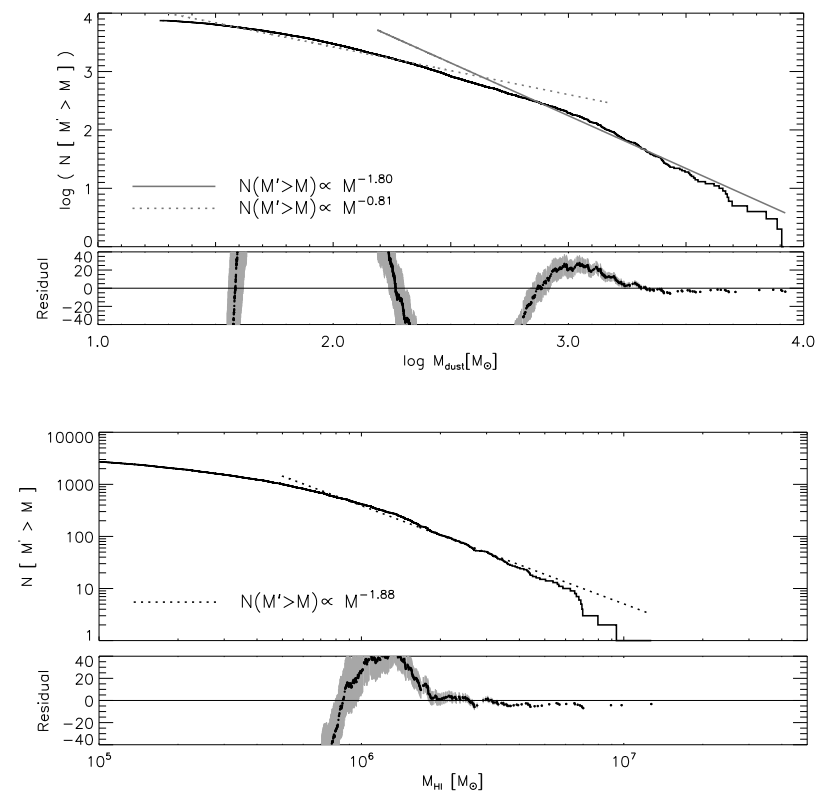

Fig. 4. Dust clump mass spectrum (Top). The slope of the mass spectrum, $\gamma$, is -1.8 with an error estimate of \pm 0.1 . H I clump mass spectrum (Bottom). The slope of the H I mass spectrum, $\gamma$, is -1.88 with an error estimate of \pm 0.1 for the fit in the selected mass range.

To characterize the clump properties, the mass spectrum of the clumps is examined. We choose the cumulative spectrum rather than the differential mass distribution since the relative masses will be more or less the same, when the mass range is small over the entire spectrum. For each catalog, we fit a power law function to the cumulative mass distribution (Rosolowsky et al. 2005; Kim et al. 2007):

$N\left(M^{\prime}>M\right)=N_{0}\left(\frac{M}{10 M_{\odot}}\right)^{\gamma+1}$,

where $N_{0}$ is the number of clouds in the derived distribution with masses higher than $10 M_{\odot}$ and $\gamma$ is the index of the differential mass distribution. The cumulative mass distribution of dust cores in the catalog of sources identified in this study and the residuals of the fit are presented in Fig. 4. The clump mass spectrum follows a broken power law in the mass distribution. For masses lower than $4 \times 10^{2} M_{\odot}$, it follows a power law distribution and decreases slowly with an exponent of the mass distribution, $\gamma=$ -0.8 . For the clumps more massive than $4 \times 10^{2} M_{\odot}$, the mass spectrum is significantly steeper with a slope of $\gamma=-1.8 \pm 0.1$ than in the lower mass regime. The observed clump mass spectrum for $1.8 \times 10^{1} M_{\odot}<M_{\text {dust }}<4.0 \times 10^{2} M_{\odot}$, exhibits a flatter power law than the stellar initial mass function (Salpeter 1955). This confirms that most of the clump mass resides in massive clumps, while the most of the stellar mass is in low-mass stars (McKee \& Williams 1997; Johnstone et al. 2000).

\subsection{Gas and dust mass spectrum}

Observation and analysis of the dust clumps and neutral cloud structure indicate that a number of smaller clumps reside in the neutral diffuse clouds. The physical properties of dust clumps were characterized in terms of their mass spectrum, which follows a power law distribution. We note that the distribution of dust clump mass is quite similar to the H I mass distribution, the index of the mass distribution being, $\gamma=-1.88$. The mass 
of the H I cloud was determined from its integrated intensity $I$ measured in $\mathrm{K} \mathrm{km} \mathrm{s}^{-1}$

$M_{\text {cloud }}=1.822 \times 10^{18} I \sigma_{\mathrm{cl}} m_{\mathrm{H}}$,

where $\sigma_{\mathrm{cl}}$ is the total cloud area in $\mathrm{cm}^{2}$. Since the dust emission in the far-IR continuum emission provides an estimate of the star formation rate (SFR) and grains remain strongly coupled to the neutral gas, we expect the mass spectra of the dust clouds to behave in a similar way to the mass spectra resulting in the diffuse gas.

The mass function near the high-end appears to be even steeper with $\gamma=-2.1$. The index of that power law fit can be steeper where star formation is ongoing, such that cloud dissipation occurs (Wada et al. 2000). The similarity of clump mass distribution suggests that the overall gas-to-dust mass ratio is more or less uniform in the region considered here. This is consistent with the overall gas-to-dust mass ratio being relatively uniform across the LMC, except for the super-shell affected by the supernova shocks (Gordon et al. 2003). A detailed analysis of the gas-to-dust mass ratio of two molecular clouds in the LMC can be found in Roman-Duval et al. (2010).

\section{Summary}

Dust clumps have been identified and cataloged in the Herschel SPIRE survey of the LMC using an automated cloud-finding algorithm. The distribution of cold dust clumps is remarkably similar to the H I clump mass distribution, sharing an index of mass distribution, $\gamma=-1.8$. However, the dust clump mass spectrum in the lower mass regime follows a flatter power law than the Salpeter stellar IMF.

Acknowledgements. We acknowledge financial support from the NASA Herschel Science Center, JPL contracts \# 1381522 \& 1381650. We thank the contributions and support from the European Space Agency (ESA), the PACS and SPIRE teams, the Herschel Science Center and the NASA Herschel Science Center (esp. A. Barbar and K. Xu) and the PACS and SPIRE instrument control centers, without which none of this work would be possible. We thank the referee for his/her very important comments on the manuscript. S.K. and E.K. were supported by Basic Science Research Program through the National Research Foundation of Korea (NRF) funded by the Ministry of Education, Science and Technology 2009-0066892. M.R. is supported by FONDECYT No. 1080335 and FONDAP No. 15010003.

\section{References}

Bergin, E. A., \& Tafalla, M. 2007, ARA\&A, 45, 339

Chi, X., \& Wolfendale, A. W. 1991, ICRC, 2, 233

Cox, P., Kruegel, E., \& Mezger, P. G. 1986, A\&A, 155, 380

Draine, B. T., \& Lee, H. M. 1984, ApJ, 285, 89

Draine, B. T., \& Li, A. 2001, ApJ, 551, 807

Gordon, K. D., Clayton, G. C., Misselt, K. A., Landolt, A. U., \& Wolff, M. J. 2003, ApJ, 594, 279

Gordon, K. D., et al. 2010, A\&A, 518, L89

Griffin, M. J., et al. 2010, A\&A, 518, L3

Ivezic, Z., Nenkova, M., \& Elitzur, M. 1997, MNRAS, 287, 799

Johnstone, D., Wilson, C. D., Moriarty-Scieven, G., et al. 2000, ApJ, 545, 327

Kim, S., Martin, P. G., \& Hendry, P. D. 1994, ApJ, 422, 164

Kim, S., Staveley-Smith, L., Dopita, M. A., et al. 1998, ApJ, 503, 674

Kim, S., Dopita, M., Staveley-Smith, L., \& Bessell, M. 1999, AJ, 118, 2797

Kim, S., Staveley-Smith, L., Dopita, M. A., et al. 2003, ApJS, 148, 473
Kim, S., Rosolowsky, E., Lee, Y., et al. 2007, ApJS, 171, 419

Mathis, J. S., Rumpl, W., \& Nordsieck, K. H. 1977, ApJ, 217, 425

McKee, C. F., \& Williams, J. P. 1997, ApJ, 476, 144

Meixner, M., Gordon, K., Indebetouw, R., et al. 2006, AJ, 132, 2268

Meixner, M., et al. 2010, A\&A, 518, L71

Pilbratt, G. L., et al. 2010, A\&A, 518, L1

Poglitsch, A., et al. 2010, A\&A, 518, L2

Roman-Duval, J., et al. 2010, A\&A, 518, L74

Rosolowsky, E. 2005, PASP, 117, 1403

Salpeter, E. E. 1955, ApJ, 121, 161

Sarkar, G., \& Sahai, R. 2006, ApJ, 644, 1171

Silva, L., Granato, G., Bressan, A., \& Danese, L. 1998, ApJ, 509, 103

Spitzer, L. 1978, Physical processes in the interstellar medium (New York: Wiley-Interscience)

Staveley-Smith, L., Kim, S., Calabretta, M., Haynes, R., \& Kesteven, M. 2003, MNRAS, 339, 87

Strong, A. W., Moskalenko, I. V., \& Reimer, O. 2000, ApJ, 573, 763

Wada, K., Spaans, M., \& Kim, S. 2000, ApJ, 540, 797

Weinberg, M. D., \& Nikolaev, S. 2001, ApJ, 548, 712

Weingartner, J. C., \& Draine, B. T. 2001, ApJ, 548, 296

Williams, J., de Geus, E. J., \& Blitz, L. 1994, ApJ, 428, 693

1 Astronomy \& Space Science, Sejong University, 143-747, Seoul, South Korea

e-mail: sek@sejong.ac.kr

2 CEA, Laboratoire AIM, Irfu/SAp, Orme des Merisiers, 91191 Gif-sur-Yvette, France

3 Space Telescope Science Institute, 3700 San Martin Drive, Baltimore, MD 21218, USA

4 Steward Observatory, University of Arizona, 933 North Cherry Ave., Tucson, AZ 85721, USA

5 Sterrewacht Leiden, Leiden University, PO Box 9513, 2300 RA Leiden, The Netherlands

6314 Physics Building, Department of Physics and Astronomy, University of Missouri-Columbia, Columbia, MO 65211, USA

7 Department of Astronomy, Lab for Millimeter-wave Astronomy, University of Maryland, College Park, MD 20742-2421, USA

8 Department of Physics and Astronomy, University College London, Gower Street, London WC1E 6BT, UK

9 Mullard Space Science Laboratory, University College London, Holmbury St. Mary, Dorking, Surrey RH5 6NT, UK

10 Centre d'Étude Spatiale des Rayonnements, CNRS, 9 avenue du Colonel Roche, BP 4346, 31028 Toulouse, France

11 Observatoire Astronomique de Strasbourg, 11 rue de 1'université, 67000 Strasbourg, France

12 Center for Supercomputing and Astrophysics, Swinburne University of Technology, Hawthorn VIC 3122, Australia

13 CSIRO Australia Telescope National Facility, 76 Epping Rd., NSW1710, Australia

14 Department of Astrophysics, Nagoya University, Chikusa-ku, Nagoya 464-8602, Japan

15 Department of Physical Science, Osaka Prefecture University, Gakuen 1-1, Sakai, Osaka 599-8531, Japan

16 Spitzer Science Center, California Institute of Technology, MS 220-6, Pasadena, CA 91125, USA

17 Max-Planck-Institut für extraterrestrische Physik, Giessenbachstra 85748 Garching, Germany

18 Stratospheric Observatory for Infrared Astronomy, Universities Space Research Association, Mail Stop 211-3, Moffett Field, CA 94035, USA

19 Center for Astrophysics, 60 Garden St., MS 67, Harvard University, Cambridge, MA 02138, USA

20 Departamento de Astronomia, Universidad de Chile, Casilla 36-D, Santiago, Chile 